\title{
Review of: "Optimizing the geodetic networks based on the distances between the net points and the project border"
}

\author{
Tomaž Ambrožič ${ }^{1}$ \\ 1 University of Ljubljana
}

Potential competing interests: The author(s) declared that no potential competing interests exist.

In the article, the authors deal with the optimization of the gteodethic networks. They define new criterion considering the distances between the net points and the project border in terms of neighboring. They determine relationship between the optimized parameters and the perpendicular distance connecting net points with project border mathematically correct. Three normalized neighboring objective functions based on L1, L2, and Lo norms were formulated to build the corresponding neighboring optimization models.

Simulated numerical results demonstrated the theoretical feasibility of neighboring models in improving the accuracy of observations and transportation costs between points, and demonstrated the capability of NL $\infty$ to provide a precise network and preserve the initial reliability and observation cost. This feasibility is required to be demonstrated in common measurements used in real applications such as angles, distances and GPS observations.

Nevertheless, it is correct that the article has been published. 